\title{
UrtheCast SECOND-GENERATION EARTH OBSERVATION SENSORS
}

\author{
Dr. Keith Beckett
}

UrtheCast Corp, Vancouver, BC, Canada - KBeckett@UrtheCast.com

KEY WORDS: Dual-mode optical camera, dual-band SAR sensor, International Space Station

\begin{abstract}
:
UrtheCast's Second-Generation state-of-the-art Earth Observation (EO) remote sensing platform will be hosted on the NASA segment of International Space Station (ISS). This platform comprises a high-resolution dual-mode (pushbroom and video) optical camera and a dual-band (X and L) Synthetic Aperture RADAR (SAR) instrument. These new sensors will complement the firstgeneration medium-resolution pushbroom and high-definition video cameras that were mounted on the Russian segment of the ISS in early 2014.
\end{abstract}

The new cameras are expected to be launched to the ISS in late 2017 via the Space Exploration Technologies Corporation Dragon spacecraft. The Canadarm will then be used to install the remote sensing platform onto a CBM (Common Berthing Mechanism) hatch on Node 3, allowing the sensor electronics to be accessible from the inside of the station, thus limiting their exposure to the space environment and allowing for future capability upgrades.

The UrtheCast second-generation system will be able to take full advantage of the strengths that each of the individual sensors offers, such that the data exploitation capabilities of the combined sensors is significantly greater than from either sensor alone. This represents a truly novel platform that will lead to significant advances in many other Earth Observation applications such as environmental monitoring, energy and natural resources management, and humanitarian response, with data availability anticipated to begin after commissioning is completed in early 2018.

\section{INTRODUCTION}

UrtheCast was born out of a novel vision to democratize Earth Observation (EO) by streaming imagery from various sensors on the ISS via the web to the consumer - a vision enabled by cost advantages enabled by using the ISS for space-based remote sensing. Without the usual power, mass and thermal constraints for a stand-alone spacecraft, it dramatically changes the economics of EO from Space.

The ISS operates in a unique orbit, unusual for remote sensing, having an inclination angle of $51.6^{\circ}$. The orbit is not sunsynchronous and precesses at a rate of roughly $6^{\circ}$ per day, giving the ability to see the earth at a wide range of sun illumination angles.

\section{FIRST-GENERATION}

The first-generation system introduces the completely novel concept of space-based Ultra High-Definition (UHD) fullmotion video (FMV) combined with wide-area surveillance to the Earth Observation market giving users the ability to visualize earth-based activity and its surrounding context.

This idea is not entirely new though. The earliest example dates back to 1972 when the first three Landsat satellites carried the Return Beam Vidicon (RBV) sensor, in addition to the MultiSpectral Scanner (MSS) sensor [1]. The RBV provided video data while the MSS provided wide-area surveillance.

The RBV was a modified television camera-like instrument that took snapshot images of the earth along the ground track of the satellite. The RBV carried by Landsat- 1 and 2 operated in three spectral bands: blue-green, orange-red and near-infrared, with a swath width of $90 \mathrm{~km}$ and $80 \mathrm{~m}$ resolution. Later, Landsat-3 carried a pair of side-by-side mounted panchromatic RBV with a combined swath width of $180 \mathrm{~km}$ and $40 \mathrm{~m}$ resolution.

Now, more than 40 years later, UrtheCast, a multi-national company based in Vancouver Canada is deploying the same philosophy. In partnership with Roscosmos (the Russian Federation Space Agency) and RSC Energia, UrtheCast is now operating two cameras installed on the Russian segment of the International Space Station, one is a wide area MediumResolution Camera (MRC) and the other is a High-Resolution Camera (HRC) that provides full motion video.

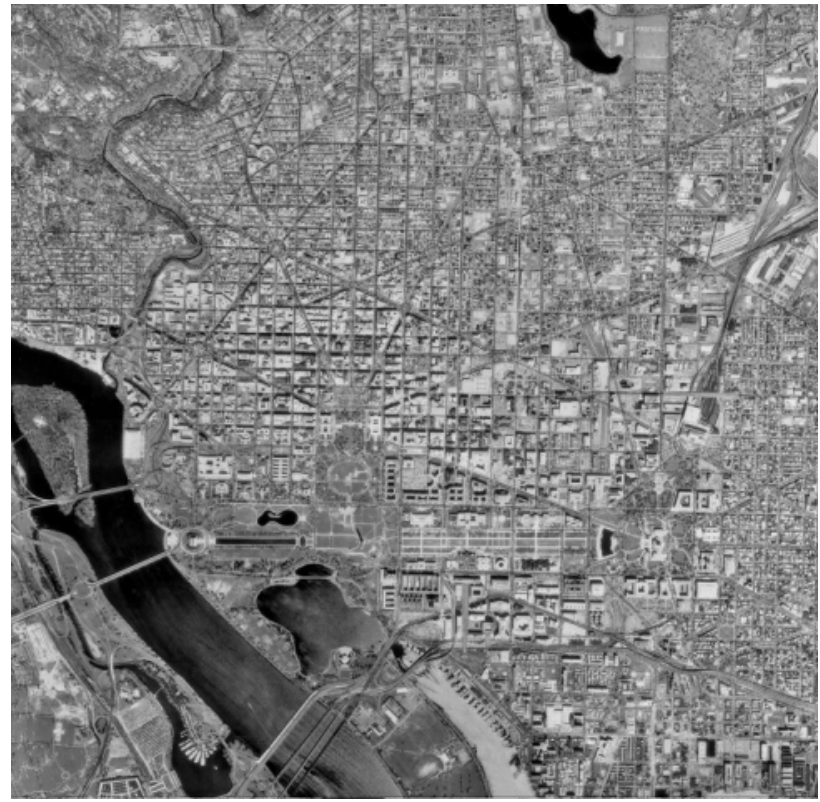

Figure 1. Example Landsat-3 RBV Image of Elko, Nevada [2] 


\subsection{Space Segment}

The MRC and HRC cameras are mounted on the starboard side of the Zvezda Service Module.

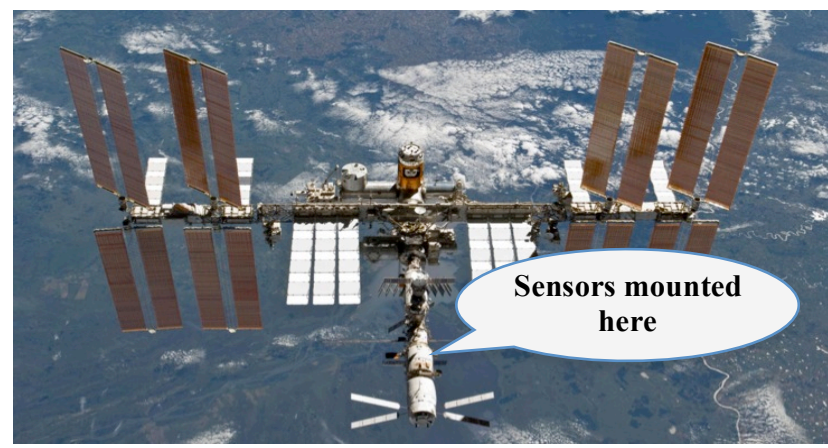

Figure 2. Location of the First-Generation Sensors on the ISS

The MRC is a $5 \mathrm{~m}$-class pushbroom sensor with a nominal $50 \mathrm{~km}$ wide swath (at nadir for $400 \mathrm{~km}$ altitude), which has four spectral channels: blue, green, red and near-infrared (NIR). The MRC is rigidly attached to the ISS in a near nadir-pointing configuration.

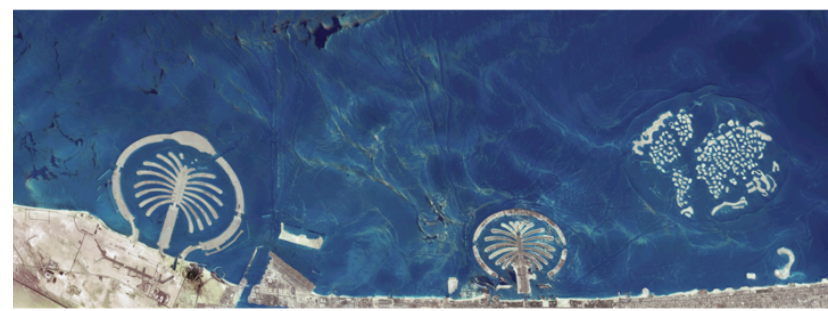

Figure 3. MRC Image of the Palm Island in Dubai

The HRC is a $1 \mathrm{~m}$-class video sensor using a 14 MPixel CMOS detector (operated at 3 Frames Per Second) with a nominal 3.8 $\mathrm{km}$ by $5.7 \mathrm{~km}$ footprint (at nadir for $400 \mathrm{~km}$ altitude) which has a Bayer filter on the detector with three spectral channels: blue, green and red. The HRC is attached to a Bi-axial Pointing Platform (BPP), steering the camera in the roll and pitch directions allowing the camera to dwell on a target for up to 90 seconds.

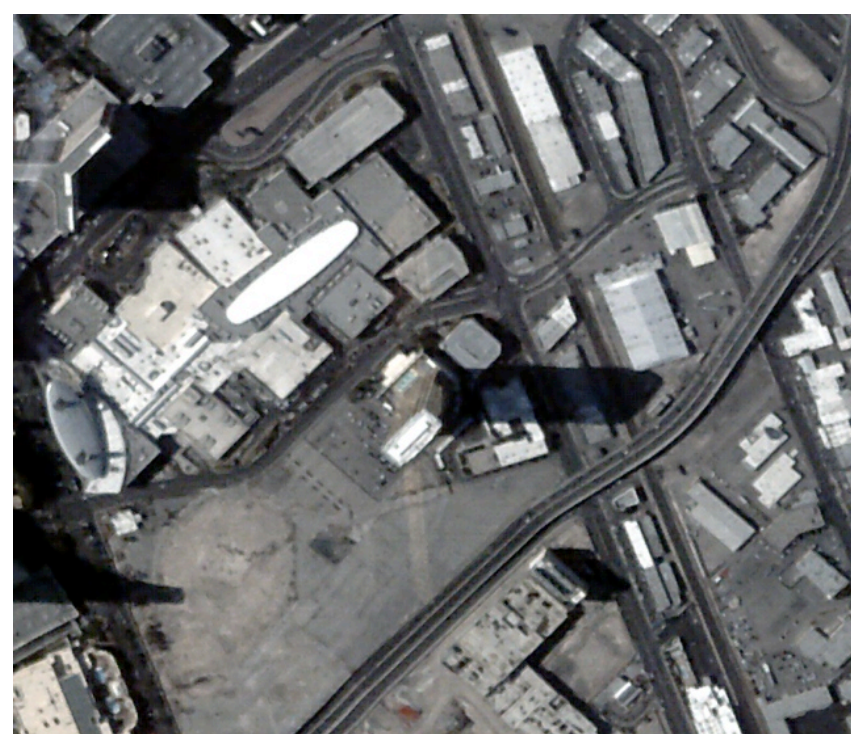

Figure 4. HRC Image of the Trump Tower in Las Vegas, Nevada

\subsection{Ground Segment}

UrtheCast has built the data receiving, archiving, processing and ordering systems to operate in the Amazon Web Services (AWS) cloud infrastructure. The Ground Segment (GS) capacity can therefore be elastically scaled up and down as the demand requires.

An industry standard set of Rapid Positioning Capability (RPC) Model and Ortho Model (OM) products and videos are currently supported. The image processing is build around a PCI Geomatics GXL/ JPS core using the Python programming language. The GS can therefore be quickly modified to offer new products and services.

\subsection{Web Platform}

UrtheCast has built the Web Platform (WP), also hosted in AWS, to provide advanced product distribution and customer experience. The WP offers additional customer-selectable postprocessing options on the imagery and video data.

\section{SECOND-GENERATION}

The second-generation system is an extension of the systems and lessons learned in the first-generation system.

\subsection{Space Segment}

In partnership with NanoRacks LLC, UrtheCast has recently secured an agreement to install a dual-mode optical camera and a dual-band SAR on the NASA Segment of the International Space Station. Both sensors are mounted onto a custom module being jointly built by UrtheCast and NanoRacks, called the Lightweight UrtheCast NanoRacks Alcove (LUNA), which is attached to the aft CBM hatch on the Tranquillity Module (node 3 of the ISS).

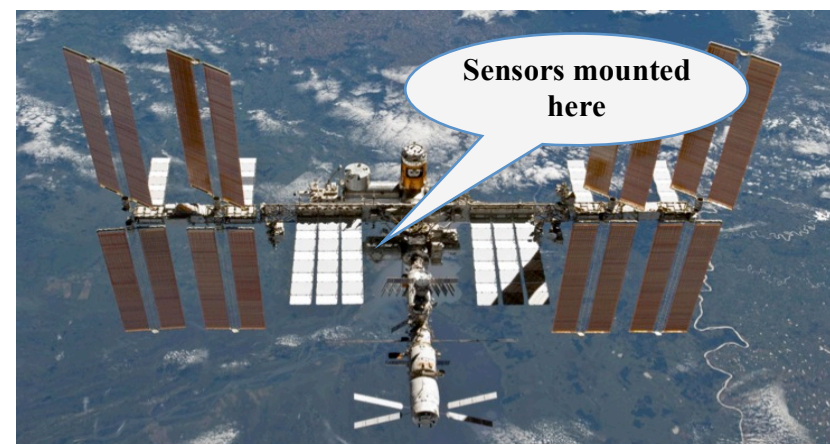

Figure 5. Location of the Second-Generation sensors on the ISS

Each sensor is deployed from the LUNA module on a dedicated boom which has its own three-axis gimbal mechanism, providing control in the roll, pitch and yaw directions, allowing the sensors to be operated independently, either dwelling on a target or covering a larger area for up to 90 seconds as the ISS passes overhead. It is possible to operate both sensors in tandem or individually, and to point both sensors at the same target area or different target areas.

The LUNA module also has its own multi-Tbit on-board data storage and wideband X-band downlink. The downlink system comprises two independent high gain antennas on individual 2axis gimbals that use left and right circular polarizations, providing a combined data rate of 1 Gbps. This is augmented with access to NASA's ISS communications system that uses 
the Tracking and Data Relay Satellite System (TDRSS), giving near-continuous uplink and downlink communication capabilities.

The $2^{\text {nd }}$ generation system is now in development with a planned launch date of Q4 2017.

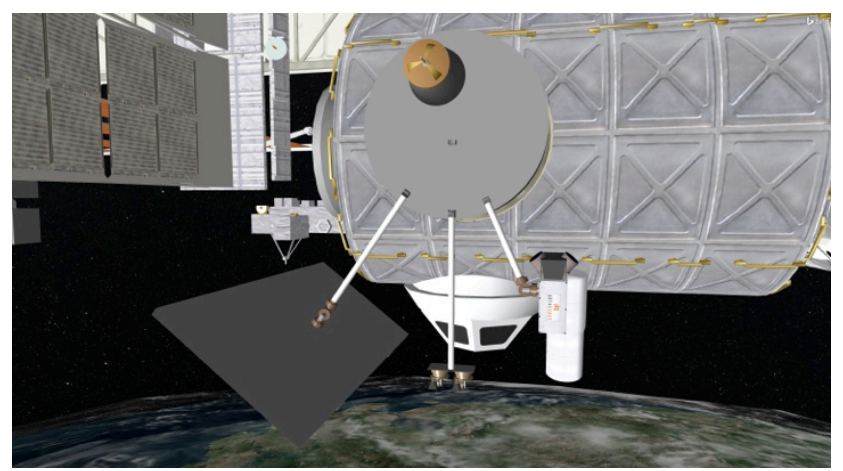

Figure 6. Close-up view of the Second-Generation sensors

\subsubsection{Dual-Mode Optical Camera}

The dual-mode camera can be operated either in pushbroom or video mode.

The pushbroom sensor uses a 64-stage Time Delayed Integration (TDI) architecture, digitized to 14 bits. This sensor yields a nominal $12 \mathrm{~km}$ swath (at nadir for $400 \mathrm{~km}$ altitude) and is comprised of a panchromatic channel giving $0.5 \mathrm{~m}$-class imagery and six multispectral channels giving $1 \mathrm{~m}$-class imagery: blue, green, yellow, red, red-edge and near-infrared (NIR).

The video sensor uses a 20 MPixel CMOS detector that yields a nominal $3.5 \mathrm{~km}$ by $2.7 \mathrm{~km}$ footprint (at nadir for $400 \mathrm{~km}$ altitude) at up to $30 \mathrm{FPS}$, digitized to 12 bits. This detector uses a Bayer filter that provides three spectral channels (red, blue and green) giving $0.5 \mathrm{~m}$-class imagery.

Augmenting the dual-mode optical camera is a meteorological camera (MetCam), providing additional spectral channels, albeit at a lower resolution, designed to measure the impact of the atmospheric on the imagery and enable its correction during onground image processing. The data from the MetCam is not included in the distributed product though.

\subsubsection{Dual-Band Synthetic Aperture RADAR}

The dual-band, L- and X-band, Synthetic Aperture RADAR (SAR) can be operated in one of three modes: SpotLight, StripMap and ScanSAR.

The SpotLight mode is able to acquire $1 \mathrm{~m}$-class (X-band) and $5 \mathrm{~m}$-class (L-band) imagery with a nominal size of $5 \mathrm{~km}$ by $5 \mathrm{~km}$.

The StripMap mode is able to acquire $2 \mathrm{~m}$-class (X-band) and $10 \mathrm{~m}$-class (L-band) imagery with a nominal swath width of $10 \mathrm{~km}$.

The ScanSAR mode is able to acquire 10m-class (X-band) and $30 \mathrm{~m}$-class (L-band) imagery with a nominal swath width of $25 \mathrm{~km}$ when both bands are operated together. When operating L-band alone, the ScanSAR mode is able to acquire $30 \mathrm{~m}$-class imagery with a swath width of up to $100 \mathrm{~km}$.
The L-band SAR supports the full complement of polarization options, including single, dual, quad, linear-compact and circular compact pole. The X-band SAR supports VV polarization only.

The SAR data can also be used to generate interferometric products.

\subsection{Ground Segment and Web Platform}

The UrtheCast GS and WP will be upgraded to support the new sensors and the standard product types. In addition, a new suite of product types and services are envisioned that leverage the information available from the Second-Generation Sensors.

\section{PRODUCTS AND SERVICES}

\subsection{Standard Products}

The Second-Generation System will generate an industry standard set of Rapid Positioning Capability (RPC) Model and Ortho Model (OM) products and videos. These products will include the imagery (all Optical sensor spectral channels and all SAR sensor polarizations) and the associated metadata for both sensors.

\subsection{Fusion Products}

The SAR and Optical data are highly complementary in terms of the information that can be extracted from each source.

The polarization and dielectric measurement provided by SAR data can support determination of material classification, wetness, structure, texture and roughness information about the scene that the Optical data often cannot. For example, agriculture and forestry applications based on Optical data need to include a correction for soil moisture. And the SAR data can also assist in differentiating plant and tree types based on their polarization information. The SAR interferometric products can also be used to measure minute variations in the earth's surface.

The spectral measurement provided by Optical data can support determination of signature classification information about the scene that SAR data often cannot. For example, the spectral signatures of different types of man-made objects, vegetation and geologic features are all well characterized in Optical data, and less so in SAR data.

The addition of a time-series of images acquired by the video sensor over the arc of acquisition geometries yields an even deeper understanding of the scene due to the 3D surface model and motion vector information that it provides.

Consequently, the fusion of Optical imagery, SAR imagery and interferometric information, 3D surface model and motion vector products yields a suite of products where there is significantly more information that can be extracted from any individual data source alone. This concept is well appreciated and it is worth noting that since 2006, the IEEE Geoscience and Remote Sensing Society (GRSS) has sponsored multiple Data Fusion Contests, several of which (2009/2010 and 2012) involved both Optical and SAR imagery [3].

With co-incident Optical and SAR imagery, 3D surface model and motion vector information, the accuracy and range of possible applications becomes even more interesting because it eliminates a variety of unaccountable sources of error, typical of 
most fusion products resulting from the misregistration due to weather conditions, solar illuminations, temporal scene changes, viewing geometries, etc.

For example, in forestry biomass estimation, the X-band SAR sensor is used to locate the tops of the trees and the L-band SAR sensor is used to locate the bottom of the tree, thus yielding an accurate stand height. The pushbroom sensor is used to perform spectral classification to determine tree species and stand density. The video sensor is used to construct a 3D surface model of the scene and correct for any errors in the intermediate results. The data is then fused, in conjunction with the appropriate forestry models, in order to estimate the biomass.

The processing of high-resolution SAR data will benefit greatly from being fused with accurate $3 \mathrm{D}$ surface models and motion vector information, thus yielding accurately focused imagery. SAR data has been traditionally processed assuming a smooth earth or a coarse DEM, and therefore tends to be somewhat out of focus and suffer from layover and shadow artefacts. Since
SAR data relies on the Doppler phase history, objects that are moving are therefore mis-located.

The cyclic nature of the solar illumination variations from the ISS orbit will allow construction of shadow-free 3D image models of cities by fusing both Optical and SAR data acquired over several orbits, giving both colour and structural information for all surfaces.

The images below illustrate a simple example of multi-sensor data fusion, combining X-band SAR, L-band SAR and multispectral Optical imagery, resulting in a very content-rich information product.

\subsection{Applications and Services}

The standard and fusion products will feed into applications and services such as data analytics, site monitoring and wide-area reconnaissance.

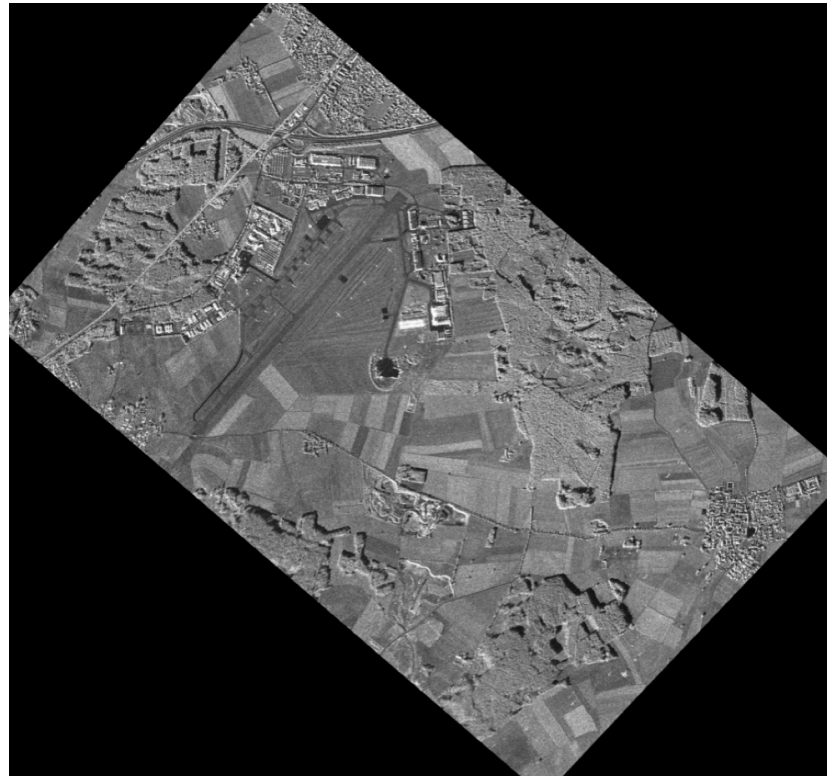

Figure 7. Sample X-band SAR image

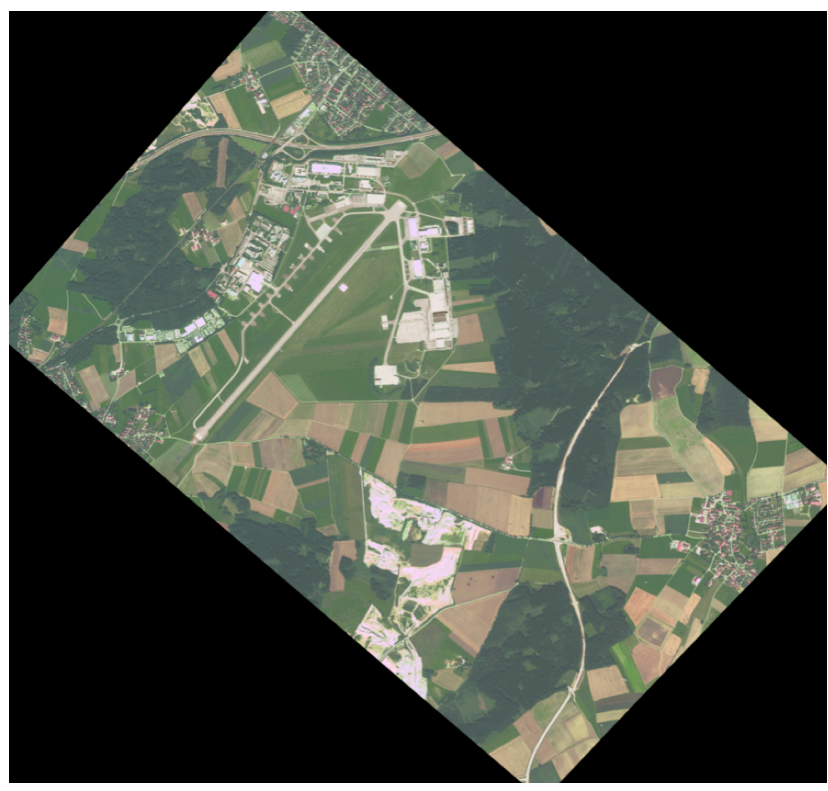

Figure 9. Sample Multispectral Optical image

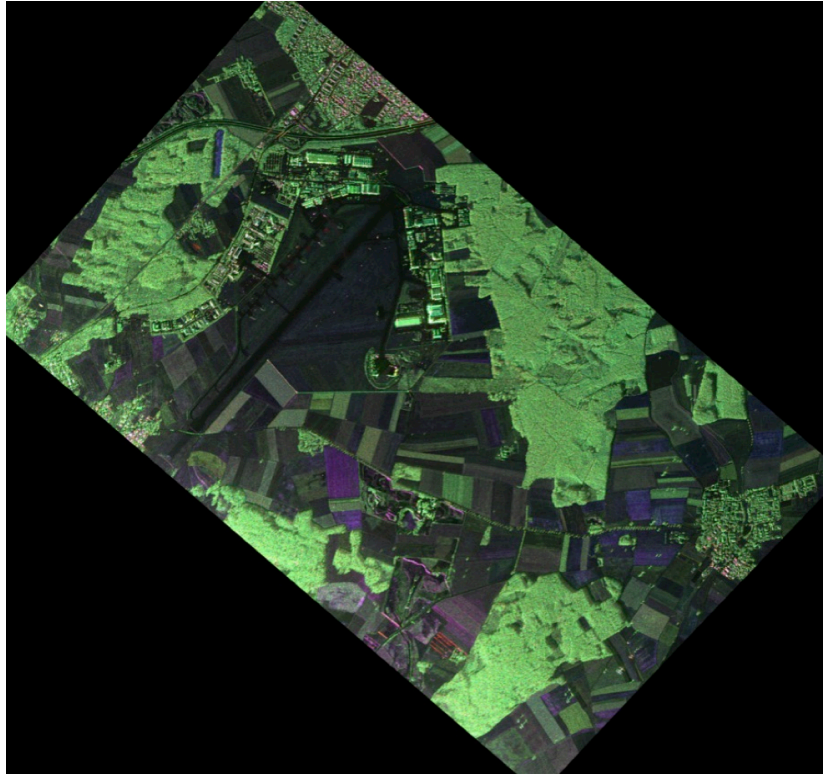

Figure 8. Sample L-band SAR image

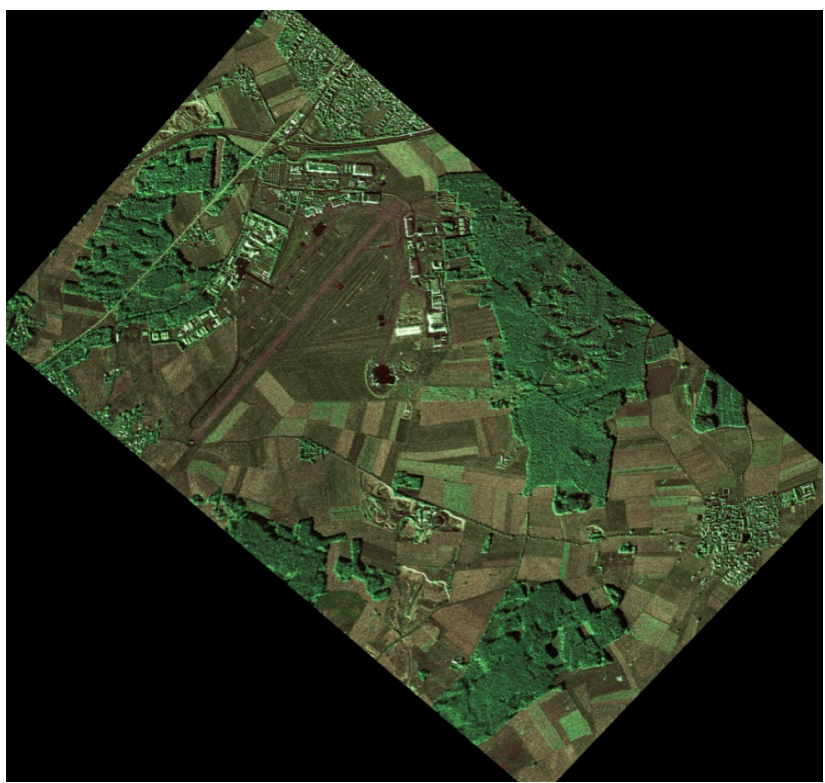

Figure 10. Sample Fused image 
The Second-Generation System will serve as a platform on for advancing the Research and Development of a host of new applications and services. The combination of on-board processing capability, flexible and highly-configurable Optical and SAR sensor acquisition modes, and near-continuous communications will be used to support experiments involving novel acquisition geometries and imagery exploitation, and reducing latency in the delivery of actionable information to end users.

Just a small subset of the potential application areas that are currently being explored are ...

Maritime Surveillance: The SAR and Optical sensors could be used in a cross-queuing scenario. The SAR sensor would be squinted forward to scan the ocean, the on-board processor would perform real-time ship detection and the Optical sensor would be commanded to look backward to acquire highresolution images of selected ships for positive identification and detection of any pollution discharges. Only the OTH-Gold (Over-The-Horizon) messages, together with their corresponding image chips from both the SAR and Optical sensor, would then need to be downlinked with no additional on-ground processing required.

Oil Spill Tracking: The X-band SAR provides superior oil-tosea contrast. The fully polarimetric L-band SAR combined with Optical data provides oil classification in terms of its makeup (plant or mineral) and thickness.

Camouflage Detection: The penetration capabilities of the SAR sensor would be exploited to detect vehicles or other manmade structures hidden beneath natural or artificial foliage. When the Optical sensor fails to detect the same vehicles or structures, this indicates a likely camouflage situation.

Decoy Detection: The material classification capabilities of the SAR sensor would be exploited to differentiate real vehicles from wood, paper or plastic decoys that can easily fool an Optical sensor.

Disturbed Earth Detection: The penetration capabilities of the SAR sensor would be exploited to detect changes to the soil. When the Optical sensor fails to detect the same disturbances, this indicates a likely recent change.

Port Monitoring: The SAR and Optical sensors would be used to count shipping containers and determine stockpile volumes.

Car Counting: The SAR sensor would be used to count parked vehicles located around factories, shopping malls, gathering places and transportation hubs. The ISS orbit would be exploited to yield car counts at constantly changing times of day, enabling monitoring of worker shifts, determining peak shopping times and tracking commuter levels.

\section{CONCLUSIONS}

The key benefits of the UrtheCast Second-Generation System are significant improvements to monitoring, change detection, situational awareness and activity characterization capabilities as compared to traditional space-based remote sensing systems.

The advantages of SAR sensors are well known, providing reliable image acquisition at any time of day or night, and any weather conditions. It is therefore possible to guarantee, as a minimum, a SAR image in case the Optical image is either cloudy or not adequately illuminated by the sun. Another advantage of SAR is that it provides texture and roughness information that characterizes the scene content.

The advantages of Optical sensors are equally well known, where the spectral information provides easy to interpret and classify imagery.

With a rich set of imagery and metadata acquired over a longer dwell time, accurate 3D model reconstruction and motion vector analysis of the scene is possible. Furthermore, this information is useful in generating accurate and higher-value products.

The UrtheCast system will give much greater context regarding the nature of the location and activities being viewed. Rather than just seeing static numbers of people, vehicles or marine traffic within the targeted area, imagery analysts can better detect temporal patterns and assess their significance in the context of the scene, and can do so in a time frame that matters to people.

In short, the Second-Generation System exemplifies the old adage of "one plus one equals three" by combining the best of both Optical and SAR sensors yielding more than can be achieved from either sensor alone. In this paper, we have begun to describe only a very small subset of the range of exciting and new applications that will be possible.

\section{REFERENCES}

[1] Richards, J. A, 2006. Remote Sensing Digital Image Analysis. Canberra, Australia, pp. 393-396.

[2] USGS Landsat Data Archive, https://lta.cr.usgs.gov/rbv.html.

[3] GRSS Data Fusion Contest, http://www.grssieee.org/community/technical-committees/data-fusion/pastdata-fusion-contests/. 